\title{
Risk factors and perinatal outcome associated with low birth weight in a prospective cohort: is there a shift towards sustainable developmental goal 3
}

\author{
Sandhyasri Panda ${ }^{1 *}$, Sai Sunil Kishore M. ${ }^{2}$, Durga Devi M. ${ }^{1}$, Mahalakshmi G. ${ }^{1}$, \\ Sirisha S. ${ }^{1}$, Maneesha Kiran ${ }^{1}$
}

${ }^{1}$ Department of Obstetrics and Gynecology, MIMS, Vizianagaram, Andhra Pradesh, India
${ }^{2}$ Department of Paediatrics, MIMS, Viziznagaram, Andhra Pradesh, India

Received: 24 August 2019

Accepted: 12 September 2019

*Correspondence:

Dr. Sandhyasri Panda,

E-mail: dr.sandhyasree@gmail.com

Copyright: () the author(s), publisher and licensee Medip Academy. This is an open-access article distributed under the terms of the Creative Commons Attribution Non-Commercial License, which permits unrestricted non-commercial use, distribution, and reproduction in any medium, provided the original work is properly cited.

\begin{abstract}
Background: Low birth weight is a socio, economic, cultural and community based health issue which reflects responsibility and commitment of local and national administrative authorities. It continues to be a cause of short and long term adverse perinatal outcome with a bearing on adult non communicable health risks.

Methods: This is a prospective observational and analytic study to know the prevalence, risk factors and perinatal outcome of LBW, from July 2017 to December 2018 in department of Obstetrics and Gynecology, MIMS Medical College, Andhra Pradesh, India. Maternal risk factors and outcomes associated with LBW were defined through risk ratios.

Results: 721 infants including $116 \mathrm{LBW}$ and $605 \mathrm{NBW}$ born during study period were included in the study. Prevalence of LBW was $16 \%$. Preterm birth accounted for 35\%, FGR for $13.8 \%$ and SGA for $51.2 \%$ of them. Maternal factors like age $<20$ years and $>35$ years, social status II to IV, below higher secondary education, house maker, primi gravida, grand multi para, BMI $<18.5 \mathrm{~kg} / \mathrm{M}^{2}$ or $>24.9 \mathrm{~kg} / \mathrm{M}^{2}, \mathrm{Hb}<11 \mathrm{gm} \%$ were having higher RR for LBW. LBW infants showed frequent association with oligo or polyhydramnious and hemorrhagic or turbid amniotic fluid. They had higher risks for non reassuring fetal heart rate changes, for induced delivery or an elective caesarean section. More often they needed NICU care for longer duration and showed a higher risk for malformations and neonatal mortality. Overall perinatal mortality was 5.54 per 1000 live birth.

Conclusions: LBW is a risk factor for neonatal morbidity and mortality; which can be minimised by institutional delivery. High prevalence PTB (35\%) warrants obstetricians to be more vigilant about indentifying the risk factors and adequate management planning. Constitutionally small baby at birth probably needs redefining normal birth weight for different ethnicity.
\end{abstract}

Keywords: FGR, LBW, Maternal risk factors, Perinatal outcome, Preterm birth, SDG 3, SGA

\section{INTRODUCTION}

Birth weight reflects socio cultural and economic empowerment of a community as well as health strategy of the local administration. Low birth weight (LBW) continues to be a key public health issue in safe motherhood projects. It causes adverse perinatal outcome and increased childhood morbidity and mortality. Nearly 
three fourth of all neonatal deaths and half of infant deaths occur among LBW infants. It is attributed as a major contributor to most of the adult onset life style diseases which pose a high risk for premature death among adults. Most importantly LBW causes recurrence of low birth weight in off springs. ${ }^{1-4}$ LBW is caused by preterm birth or birth of a growth restricted baby. Ever since Barker hypothesized foetal origin of adult diseases, it has been a field of research to change the womb environment which later determines the world environment for the baby. In India, of the 26 million born every year, 8 million are LBW, i.e. around $40 \%$ of the global burden of LBW infants. Nearly three fourth of all neonatal deaths and half of infant deaths occur among LBW infants. An LBW baby is at higher risk of both mortality and morbidity compared to the normal birth weight infant. ${ }^{2,5}$ Low birth weight (LBW) is defined by the World Health Organization (WHO) as weight at birth less than 2500g and small for gestational age (SGA) as birth weight less than tenth percentile for that gestational age in the general population; Overall, it is estimated that $15 \%$ to $20 \%$ of all births worldwide are LBW. ${ }^{2,3,6}$ Among regions, South Asia has the highest incidence of low birth weight, with one in four newborns weighing less than 2,500grams, approximating at $28 \%$. $^{3}$ The goal is to achieve a $30 \%$ reduction of the number of infants born with a weight lower than $2500 \mathrm{~g}$ by the year $2025 .^{3}$

LBW is associated with either preterm birth (PTB) i.e birth before $37+^{\circ}$ weeks , fetal growth restriction (FGR) i.e birth weight $<10^{\text {th }}$ percentile for the gestational age with either Doppler or amniotic fluid changes or, constitutionally small babies (SGA) i.e birth weight $<10^{\text {th }}$ percentile for the gestation and without pathological changes. Often associated with intrauterine asphyxia, scanty and meconium stained amniotic fluid, intrapartum asphyxia, heart rate changes and low apgar score at birth, followed by need for NICU admission and further management due to its associated morbidity and at times mortality, it adds to the cost of pregnancy and mars the concept of safe motherhood goal.

The aim of this study to know the prevalence, risk factors and perinatal outcome of LBW and to study whether there is any positive shift in the outcome as we reach towards 2025 .

To estimate the prevalence and antenatal risk factors associated with LBW, to consider labour outcomes like (spontaneous, or induced labour and elective caesarean) mode of delivery like (normal vaginal, instrumental and caesarean), indications for caesarean, the association with abnormal amniotic fluid and fetal heart rate variability in LBW. To study the perinatal outcomes like mean birth weight, mean gestational age, $1 \mathrm{~min}$ and $5 \mathrm{~min}$ apgar score, gender association and neonatal admission to NICU and the neonatal complications associated with LBW in relation to that with normal birth weight (NBW) neonates.

\section{METHODS}

This prospective analytic and observational study was undertaken in a cohort of pregnant women attending a teaching medical institution of eastern Andhra Pradesh, India for delivery during July 2017 and December 2018.

\section{Inclusion criteria}

- Booked antenatal women in this institute who undertook 4-8 visits before confinement. They were interviewed for a detailed history and their antenatal data were scrutinised at the time of admission to delivery after obtaining written informed consent for participating in the study.

\section{Exclusion criteria}

- Deliveries of anomalous babies, intrauterine deaths, still births and multiple gestations, babies with extreme prematurity ( $<28$ weeks GA) and unbooked mothers.

We have considered delivery at $<37+^{\circ}$ weeks as Preterm Birth. Less than 34 weeks as early PTB and Between $34^{+1}$ to $36^{+6}$ as late PTB.

We defined FGR neonate as a growth restricted foetus (estimated fetal weight <tenth percentile for the gestational age) associated with either oligohydramnious defined as Amniotic fluid index $<5 \mathrm{~cm}$ and/or Doppler changes or neonatal pathological changes consistent with FGR; SGA being defined as a growth restricted foetus without the former changes.

We defined abnormal amniotic fluid as either scanty (AFI $<5 \mathrm{~cm}$ ) or excessive (AFI $>18 \mathrm{~cm}$ ) liquor, meconium stained, hemorrhagic or turbid liquor. Parameters associated with LBW (birth weight <2500gms) were correlated with NBW (birth weight $>2500 \mathrm{mgs}$ ).

\section{Statistical analysis}

Statistical analysis were made by use of 2- way contingency table at $95 \%$ confidence level for RR and CI values in bivariate analysis; use of pearson chi2 test to find $\mathrm{p}$ value for test of significance for all categorical data and the student - $t$ test was used for the continuous data. $\mathrm{P}$ value $<0.05$ is considered significant.

\section{RESULTS}

Total number of deliveries during study periods were 745 (311vaginal delivery +434 caesarean delivery). Anomalous (4), still born and IUD (7), twin (12) and extreme prematurity (1); total 24 infants were excluded. We included 721 (299 VD+ 422 CD) infants in the study. Among them (10 early PT+18 late PT +55 term) $83 \mathrm{CD}+$ (6 early PT +7 late PT +20 term) $33 \mathrm{VD}=116$ were $\mathrm{LBW}$ and 605 were NBW babies. Prevalence of LBW was $16 \%$ 
considering all live births $>28$ weeks. Among them 41 (35\%) were due to PTB, $16(13.8 \%)$ were due to FGR and $59(51.2 \%)$ infants were SGA (constitutionally small baby) (Figure 1).

Table 1, Bivariate analysis showed maternal age $<20$ years (RR 1.1; 95\% CI 0.56-1.24) and >35 years (RR 2.3; 95\% CI 0.77-4.45) were associated with LBW. Home makers had more risk of LBW (RR1.33; 95\%CI0.872.05). Class I (RR 0.84; 95\% CI 0.56-1.24) and higher maternal education (RR 0.79 ; 95\% CI 0.55-1.33) were protective; LBW was seen more often among primi (RR1.18; 95\% CI 0.83-1.68) and grand multiparae (RR2.1; 95\%CI 0.56-4.46), anaemic, low BMI (RR 1.17; 95\% CI 0.75-1.78) and high BMI (RR 2.08; 95\% CI 0.10-
5.53) mothers. Anemia of increasing severity showed increasing RR towards LBW.

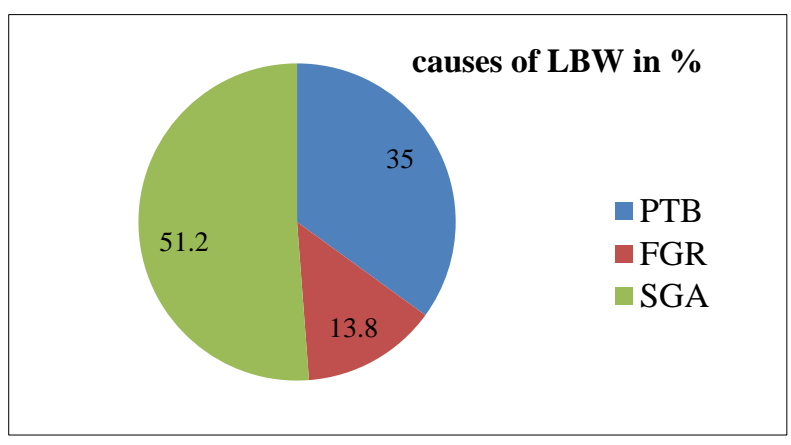

Figure 1: Causes of LBW.

Table 1: Antenatal risk factors associated with LBW.

\begin{tabular}{|c|c|c|c|}
\hline Parameters & LBW (n=116) (\%) & NBW $(n=605)(\%)$ & $\operatorname{RR}(95 \% \mathrm{CI})$ \\
\hline \multicolumn{4}{|l|}{ Age } \\
\hline$<20$ & $26(22.4)$ & $124(20.5)$ & $1.10(0.44-1.20)$ \\
\hline $21-34$ & $86(74.1)$ & $474(78.3)$ & $0.82(0.56-1.24)$ \\
\hline$>35$ & $04(3.5)$ & $007(1.2)$ & $2.3(0.77-4.45)$ \\
\hline \multicolumn{4}{|l|}{ SES } \\
\hline I & $31(26.7)$ & $187(30.9)$ & $0.84(0.56-1.24)$ \\
\hline II & $69(59.5)$ & $346(51.2)$ & $1.08(0.76-1.5)$ \\
\hline III & $13(11.2)$ & $063(10.4)$ & $1.07(0.59-1.8)$ \\
\hline IV & $03(2.6)$ & $009(1.5)$ & $1.57(0.41-3.68)$ \\
\hline \multicolumn{4}{|l|}{ Occupation } \\
\hline $\mathrm{HM}$ & $90(77.6)$ & $431(71.24)$ & $1.33(0.87-2.05)$ \\
\hline Skilled job & 15 (12.9) & $091(15.04)$ & $0.86(0.49-1.43)$ \\
\hline Unskilled labor & $11(9.5)$ & $083(13.72)$ & $0.69(0.36-1.26)$ \\
\hline \multicolumn{4}{|l|}{ Education } \\
\hline Nil & $07(6.0)$ & $026(4.3)$ & $1.33(0.59-2.57)$ \\
\hline Primary & $09(7.8)$ & $043(7.1)$ & $1.08(0.53-1.99)$ \\
\hline Secondary & $52(44.8)$ & $245(40.5)$ & $1.16(0.81-1.64)$ \\
\hline Higher secondary and college & $48(41.4)$ & $291(48.1)$ & $0.79(0.55-1.33)$ \\
\hline \multicolumn{4}{|l|}{ Parity } \\
\hline Primi & $56(48.3)$ & $261(43.1)$ & $1.18(0.83-1.68)$ \\
\hline G2-4 & $57(49.1)$ & $338(55.9)$ & $0.79(0.56-1.13)$ \\
\hline G5 and above & $03(2.6)$ & $006(1.0)$ & $2.1(0.56-4.46)$ \\
\hline \multicolumn{4}{|l|}{ BMI } \\
\hline$<18.5$ & $24(20.7)$ & $107(17.7)$ & $1.17(0.75-1.78)$ \\
\hline $18.5-24.9$ & $85(73.3)$ & $473(78.2)$ & $0.80(0.54-1.19)$ \\
\hline $25-29.9$ & $06(5.2)$ & $023(3.8)$ & $1.3(0.53-2.61)$ \\
\hline$>30$ & $01(0.8)$ & $002(0.3)$ & $2.08(0.10-5.53)$ \\
\hline \multicolumn{4}{|l|}{$\mathbf{H b} \%$} \\
\hline$\geq 11$ & $54(46.6)$ & $330(54.5)$ & $0.76(0.53-1.08)$ \\
\hline $9-10.9$ & $57(49.1)$ & $262(43.3)$ & $1.21(0.85-1.72)$ \\
\hline $7-8.9$ & $05(4.3)$ & $012(2.0)$ & $1.86(0.70-3.66)$ \\
\hline$<7$ & & $001(0.2)$ & \\
\hline
\end{tabular}

Table 2, showed that with respect to labour outcomes, abnormal amniotic fluid environment (oligo/polyhydramnious and hemorrhagic/turbid) was more frequently associated with LBW though meconium staining was more often seen among NBW infants. LBW infants were often associated with fetal heart rate abnormalities like tachycardia, bradycardia and late 
decelerations. LBW infants were frequently delivered by elective CD (RR1.77, 95\% CI 1.08-2.99) or induced labour (RR 2.03, 95\% CI 1.03-3.93) than spontaneous labour. With respect to mode of delivery LBW infants had caesarean delivery (at RR $1.98 ; 95 \%$ CI 1.27-2.95) and instrumental vaginal delivery (at RR 1.87; 95\%CI 0.66-4.41) more frequently. APH at (RR 3.56, 95\% CI 1.42-5.55) and anomalous presentation, preeclampsia and fetal distress all were at higher RR to undergo $\mathrm{CD}$ as compared to NBW infants.

Table 2: Crude estimates of risks for labour and delivery outcomes according to birth weight.

\begin{tabular}{|c|c|c|c|}
\hline Parameters & LBW (n=116) (\%) & NBW $(n=605)(\%)$ & $\mathbf{R R}(95 \% \mathrm{CI})$ \\
\hline \multicolumn{4}{|l|}{ AF Volume } \\
\hline Normal & $87(75)$ & $488(80.6)$ & 1.00 \\
\hline Oligohydramnious & $27(23.28)$ & $113(18.7)$ & $1.27(0.83-1.90)$ \\
\hline Polyhydramnious & $2(1.72)$ & $4(0.7)$ & $2.20(0.39-5.15)$ \\
\hline \multicolumn{4}{|l|}{ Amniotic fluid (AF) } \\
\hline Clear & $96(82.7)$ & $521(86.1)$ & 1.00 \\
\hline Meconium Stained & $11(9.5)$ & $69(11.4)$ & $0.88(0.46-1.58)$ \\
\hline Hemorrhagic & $5(4.3)$ & $8(1.3)$ & $2.47(0.95-4.50)$ \\
\hline Turbid & $4(3.5)$ & $7(1.2)$ & $2.33(0.78-4.54)$ \\
\hline \multicolumn{4}{|l|}{ Fetal heart rate } \\
\hline Normal & $92(79.3)$ & $504(83.3)$ & 1.00 \\
\hline Tachycardia & $7(6.0)$ & $32(5.3)$ & $1.12(0.49-2.21)$ \\
\hline Early/variable deceleration & $5(4.3)$ & $24(4.0)$ & $1.07(0.39-2.34)$ \\
\hline Late deceleration/bradycardia & $12(10.3)$ & $45(7.4)$ & $1.34(0.73-2.28)$ \\
\hline \multicolumn{4}{|l|}{ Onset of labor } \\
\hline Spontaneous & $27(23.3)$ & $223(36.8)$ & 1.00 \\
\hline Induced & $18(15.5)$ & $128(21.2)$ & $2.03(1.03-3.93)$ \\
\hline Elective C Section & $71(61.2)$ & $254(42)$ & $1.77(1.08-2.99)$ \\
\hline \multicolumn{4}{|l|}{ Mode } \\
\hline VD & $28(24.1)$ & $245(40.5)$ & 1.00 \\
\hline $\mathrm{CS}$ & $83(71.6)$ & $339(56)$ & $1.98(1.27-2.95)$ \\
\hline Instrumental & $5(4.3)$ & $21(3.5)$ & $1.87(0.66-4.41)$ \\
\hline \multicolumn{4}{|l|}{ Indication for CS } \\
\hline APH & $5(6.0)$ & $4(1.2)$ & $3.56(1.42-5.55)$ \\
\hline Anomalous presentation & $8(9.6)$ & $9(2.7)$ & $3.06(1.52-4.82)$ \\
\hline HDP & $18(21.8)$ & $24(7.0)$ & $2.97(1.87-4.3)$ \\
\hline CTG changes & $24(29.0)$ & $59(17.5)$ & $2.0(1.3-2.96)$ \\
\hline Oligohydramnious & $20(24.0)$ & $62(18.3)$ & $1.62(1.01-2.48)$ \\
\hline Diabetes & $0(0.0)$ & $18(5.3)$ & - \\
\hline CPD & $4(4.8)$ & $61(18.0)$ & $0.36(0.115-0.95)$ \\
\hline Rpt CS & $4(4.8)$ & $102(30.0)$ & $0.20(0.06-0.55)$ \\
\hline FGR & $16(13.8)$ & $08(1.32)$ & $4.65(3.00-6.05)$ \\
\hline
\end{tabular}

Table 3, LBW infants had RR5.8 of being born preterm and $\mathrm{P}=0.001$ for mean birth weight. Low APGAR score at $1 \mathrm{~m}$ and $5 \mathrm{~m}$ were significantly high among LBW infants. There was no significant gender difference between LBW and NBW infants, but a significant difference as preterm and term births were concerned. LBW infants had a low APGAR score at first and fifth minute. There was a significant risk of admission to and prolonged NICU stay for LBW $(\mathrm{P}=0.001)$; this was higher for preterm LBW than term LBW though not significant statistically $(\mathrm{P}=0.07)$. Looking into causes of NICU admission, LBW infants scored higher than NBW in all parameters except meconium aspiration syndrome and hypocalcaemia. HMD and neonatal death were seen among LBW infants, especially when PT. Hyperbilirubinemia was significantly high among PT and LBW infants and being pretern increased the risk by 3.8tims than NBW infants. Congenital deformity was seen more frequently with LBW in a risk ratio of 3.8 times. Birth asphyxia was associated with LBW infants with RR of 2.99 times than NBW infants. 
Table 3: Crude estimates of risks for perinatal outcomes according to birth weight.

\begin{tabular}{|c|c|c|c|c|c|}
\hline \multirow{2}{*}{ Parameters } & \multicolumn{2}{|c|}{ LBW (116) } & \multicolumn{2}{|c|}{ NBW (605) } & \multirow{2}{*}{$P$ value } \\
\hline & $\mathrm{n}(\%)$ & wt & $\mathrm{n}(\%)$ & wt & \\
\hline \multicolumn{6}{|l|}{ Mean birth weight } \\
\hline Preterm & $41(35)$ & $1.83 \pm 0.39$ & $21(3.5)$ & $2.84 \pm 0.294$ & 0.001 \\
\hline Term & $75(65)$ & $2.23 \pm 0.175$ & $584(96.5)$ & $2.99 \pm 0.39$ & 0.001 \\
\hline \multicolumn{6}{|l|}{ GA } \\
\hline Early PT < 34weeks & $16(13.8$ & & 00 & & 0.000 \\
\hline Late PT < 37weeks & $25(21.6$ & & $21(3.5)$ & & 0.001 (RR 5.81) \\
\hline Term $\geq 37$ weeks & $75(64.6$ & & $584(96.5)$ & & 0.001 \\
\hline \multicolumn{6}{|l|}{ Apgar score } \\
\hline 1 minute $<7$ & $26(22.4$ & & $16(2.6)$ & & 0.001 \\
\hline 5 minute $<7$ & $14(12.0$ & & $6(0.9)$ & & 0.001 \\
\hline \multicolumn{6}{|l|}{ Gender } \\
\hline Male & $62(53.4$ & & $319(52.7)$ & & 0.887 \\
\hline Preterm & $22(35.5$ & & $7(2.2)$ & & \multirow{3}{*}{0.001} \\
\hline Term & $40(64.5$ & & $312(97.8)$ & & \\
\hline Female & $54(46.6$ & & $286(47.3)$ & & \\
\hline \multicolumn{6}{|l|}{ NICU admission } \\
\hline Yes & $53(45.7$ & & $70(11.6)$ & & \multirow{2}{*}{0.001} \\
\hline No & $63(54.3$ & & $535(88.4)$ & & \\
\hline Preterm & $27(51)$ & & $4(5.7)$ & & \multirow{2}{*}{0.001} \\
\hline Term & $26(49)$ & & $66(94.3)$ & & \\
\hline \multicolumn{6}{|l|}{ Days of Hospital stay } \\
\hline $\begin{array}{l}\text { Mean days of NICU } \\
\text { stay }\end{array}$ & $9.11 \pm 6.3$ & & $4.24 \pm 1.98$ & & 0.001 \\
\hline Preterm & $11.0 \pm 07$ & & $3.75 \pm 2.75$ & & \multirow{2}{*}{0.07} \\
\hline \multirow[t]{2}{*}{ Term } & $7.15 \pm 4.4$ & & $4.27 \pm 2.75$ & & \\
\hline & $\mathbf{P T}+\mathbf{T e}$ & & PT+ Term & & \\
\hline MAS & $0+3=3$ & & $1+6=7$ & & 0.228 \\
\hline Hyperbilirubinemia & $9+13=22$ & & $2+48=50$ & & 0.000 (RR 3.83, CI 1.88-5.19) \\
\hline Hypoglycaemia & $0+2$ & & $0+1=1$ & & 0.017 \\
\hline Hypocalcemia & $1+0$ & & $1+0=1$ & & 0.191 \\
\hline HMD & $5+1=6$ & & 0 & & 0.000 \\
\hline Sepsis & $4+4=8$ & & $0+4=4$ & & 0.000 \\
\hline Cong. deformity & $0+3=3$ & & $0+2=2(\mathrm{CH}$ & Hirsprung) & 0.000 (RR 3.80, CI 1.06-5.96) \\
\hline Neonatal death & $3+0=3$ & $25.86 / 1000$ & $1 \quad 1.65 / 1$ & & 0.001 \\
\hline Birth asphyxia & 10 & & 12 & & 0.000 (RR 2.99, CI 1.60-4.61) \\
\hline
\end{tabular}

\section{DISCUSSION}

Prevalence of LBW in our study was $16 \%$. In a study by Chaudhury AK, LBW was $36.2 \%$; Coutinho et al in their large historical cohort study found it as $14.4 \%$; Manjur Kader et al, studied from data of NFHS 3 as $20.3 \% .^{7-9}$ Aother study by Mitao $\mathrm{M}$ et al, the incidence of LBW was $10.6 \% .^{10}$ Present study found that preterm birth contributed to $35 \%$ of LBW, FGR $(13.8 \%)$ and SGA $(51.2 \%)$ neonates. In their study Sangamam reported preterm (gestational age $<38$ weeks) as $39.56 \%$, IUGR as $45.97 \%$ and constitutionally small baby as $11.4 \% .^{11}$ Present study, revealed among LBW infants Male: Female as 53.4\%: $46.6 \%$; among all male infants $16.2 \%$ were LBW and among all female infants $15.8 \%$ were
LBW. In their study Chaudhury $\mathrm{AK}$ found $\mathrm{M} / \mathrm{F}$ ratio as $34.7 \%$ and $37.7 \%$ respectively. ${ }^{7}$ LBW runs a marginal high risk of being female. ${ }^{8}$ Present study revealed that socio economic class I, skilled or unskilled workers, higher education, normal BMI and absence of anemia, gravida 2-4 and maternal age 20-34 years protected against LBW, similar to study by Mitao $\mathrm{M}$ et al, and Chakraborty $\mathrm{P}$ et al. ${ }^{10,12}$ Skilled and unskilled job protected in 14\%-31\% against LBW. Therefore some type of maternal autonomy was protective against LBW. Silvestrin $\mathrm{S}$ et al, reported higher maternal education was $33 \%$ protective against LBW, which is $21 \%$ in the present study. ${ }^{13}$ Anemia was prevalent among $53.4 \%$ mothers of LBW infants and normal $\mathrm{HB} \%$ gave $24 \%$ protection against LBW. It was $43.3 \%$ in a study by sangamam R. ${ }^{11}$ 
Amniotic fluid abnormality excluding meconium staining and ominous foetal heart rate patterns were associated with LBW infants stressing upon the prevailing prematurity. ${ }^{8,6}$ Present study reinforced the observation made by other researchers. ${ }^{8}$ Present study showed an increased risk of elective $\mathrm{CD}$ and induced labours among LBW infants similar to studies made before. ${ }^{8,10}$ LBW infants had apgar score $<7$ at $1 \mathrm{~min}$ and $5 \mathrm{~min}$ in the present study similar to that of. , $^{8,11}$ Looking at indications for $\mathrm{CD}$ among $\mathrm{LBW}$ infants, ante partum hemorrhage, anomalous presentation, preeclamsia and fetal distress in form of ominous CTG changes and oligohydramnious stood at higher risk than NBW counterparts which is similar to previous studies. ${ }^{8,10}$ Maternal diabetes, CPD and repeat caesarean as indications for CD were seen at lower risk among LBW infants. $^{8}$

Considering perinatal morbidity on MAS there was no difference between LBW and NBW infants, in compliance with the observation that LBW infants were protected against MSL which can be explained by high proportion of PTB among LBW. But association with hemorrhagic and turbid liquor indicating intra uterine infection was reflected with a extreme significant difference in LBW infants being diagnosed with sepsis ( $p$ $=0.000)$. Hyperbilirubinemia was significantly high among LBW infants, HMD was exclusively a disease among LBW infants in this study. Birth asphyxia can be due to prematurity, intra uterine asphyxia as reflected by heart rate abnormalities and sepsis. All three factors had a statistical significance among the LBW infants and this was reflected by a significant risk of birth asphyxia among LBW infants in present study.

Congenital malfomations are risk factors for LBW. Present study high lights this by (RR of 3.8) extreme significane $(\mathrm{P}=0.000)$ for association with birth defects among LBW infants similar to observation by Coutinho PR et al. ${ }^{8}$

Neonatal death rate was 25.86/1000 among LBW and 5.54/ 1000 among all newborns in the present study. Sangamam reported a NMR of $53.2 \%$ among 920 LBW infants. ${ }^{11}$ The Indian data is $28 \%$ considering all newborns. ${ }^{14}$ Present study shows an improved neonatal care had reduced the perinatal mortality rate. Therefore, this study reaffirms the benefit of institutional delivery where NICU care can prevent many perinatal deaths among high risk newborns and reassures of a progressive achievement of United Nations' SDG 3 goal of reducing perinatal death to $12 / 1000$ live births. ${ }^{15}$

\section{CONCLUSION}

LBW is a risk factor for neonatal morbidity and mortality; which can be minimised by institutional delivery. High prevalence PTB (35\%) warrants obstetricians to be more vigilant about indentifying the risk factors and adequate management planning.
Constitutionally small baby at birth probably needs redefining normal birth weight for different ethnicity.

\section{ACKNOWLEDGMENTS}

Authors would like to thank profusely to all participants, all colleagues and postgraduates who cooperated for the study. Author's special gratitude goes to MIMS administration and community medicine department for their constant help for the study design and data processing.

Funding: No funding sources

Conflict of interest: None declared

Ethical approval: The study was approved by the Institutional Ethics Committee

\section{REFERENCES}

1. Goldenberg RL, Culhane JF. Low birth weight in the United States. Am J Clin Nutr. 2007;85(S):584-90.

2. Dayanithi M. Low birth weight and premature births and their associated maternal factors. Int $\mathbf{J}$ Community Med Public Health. 2018;5:2277-85.

3. World Health Organization. Global nutrition targets 2025: low birth weight policy brief (WHO/NMH/NHD/14.5). Geneva: World Health Organization; 2014. Available at: http://www.who.int/nutrition/topics/globaltargets_lo wbirthweight_policybrief.pdf. Accessed on $15^{\text {th }}$ February 2019.

4. UNICEF Data: Monitoring the situation of children and women: Low birth weight October 2014; Undernourishment in the womb can lead to diminished potential and predispose infants to early death; 2014. Available at: https://data.unicef.org/topic/nutrition/low birthweight. Accessed on $10^{\text {th }}$ February 2019.

5. Census of India. Estimates of mortality Indicators; 2014. Available

at: www.censusindia.gov.in/vital_statistics/SRS.2012/11 _chap_4 2012. Accessed on 10 ${ }^{\text {th }}$ April 2019.

6. Gabbe SG. Obstetrics normal and problem pregnancies. Elsevier, First South east edition, Chapter 33, Intra uterine Growth Restriction. 2017:738-739,743.

7. Chaudhury AK, Chaudhury A, Tiwari SC, Dwivedi R. Factors associated with low birth weightamong new borns in an urban slum community in Bhopal. Indian J Pub Health. 2013;57:20-3.

8. Coutinho PR, Cecatti JG, Surita FG, Costa ML, Morais SS. Perinatal outcomes associated with low birth weight in a historical cohort. Repro Health. 2011;8(1):18.

9. Kader M, Nirmala KP, Perera P. Socio-economic and nutritional determinants of low birth weight in India. N Am J Med Sci. 2014;6(7):302-8.

10. Mitao M, Philemon R, Obure J, Mmbaga BT, Msuya $\mathrm{S}$, Mahande MJ. Risk factors and adverse perinatal outcome associated with low birth weight in 
Northern Tanzania: a registry-based retrospective cohort study. Asian Pacific J Repro. 2016;5(1):75-9.

11. Sangamam R. Perinatal mortality and morbidity among low birth weight babies. Int J Community Med Public Health. 2015;2(1):51-8.

12. Chakraborty P, Anderson AK. Maternal autonomy and low birth weight in India. J Women's Health (Larchmt). 2011;9(20):1373-82.

13. Silvestrin S, Silva CH. Maternal Education level and low birth weight: ameta analysis. J Pediatr (Rio J). 2013;89(4):339-45.

14. Sankar MJ, Neogi SB, Sharma J, Chauhan M, Srivastava R, Prabhakar PK, et al. State of newborn health in India. J Perinatol. 2016;36(s3):S3.
15. Sustainable Development Goal. Published by: NHP Admin, created/validated by: NHP Admin, last updated by; 2018. Available at: www.nph.gov.in. Accessed on $15^{\text {th }}$ August 2019.

Cite this article as: Panda S, Kishore MSS, Devi MD, Mahalakshmi G, Sirisha S, Kiran M. Risk factors and perinatal outcome associated with low birth weight in a prospective cohort: is there a shift towards sustainable developmental goal 3. Int J Reprod Contracept Obstet Gynecol 2019;8:3858-64. 